\title{
Largeness and SQ-universality of cyclically presented groups
}

\author{
Gerald Williams
}

September 2, 2010

\begin{abstract}
Largeness, SQ-universality, and the existence of free subgroups of rank 2 are measures of the complexity of a finitely presented group. We obtain conditions under which a cyclically presented group possesses one or more of these properties. We apply our results to a class of groups introduced by Prishchepov which contain, amongst others, the various generalizations of Fibonacci groups introduced by Campbell and Robertson. Using the techniques developed we give a new, purely group-theoretic, proof of the (almost complete) classification of the finite Cavicchioli-HegenbarthRepovš groups.
\end{abstract}

Keywords: cyclically presented group, largeness, SQ-universality.

MSCs: 20E05, 20E06, 20F05.

\section{Introduction}

Let $w=w\left(x_{0}, \ldots, x_{n-1}\right)$ be a word in the free group $F_{n}$ with generators $x_{0}, \ldots, x_{n-1}$ and let $\theta: F_{n} \rightarrow$ $F_{n}$ be the automorphism of $F_{n}$ given by $\theta\left(x_{i}\right)=x_{i+1}$ for each $0 \leq i \leq n-1(\operatorname{subscripts} \bmod n)$. Define

$$
G_{n}(w)=\left\langle x_{0}, \ldots, x_{n-1} \mid w, \theta(w), \ldots, \theta^{n-1}(w)\right\rangle .
$$

Then $G_{n}(w)$ is said to be a cyclically presented group and the above presentation is said to be a cyclic presentation.

Cyclically presented groups may be trivial, finite and nontrivial, or infinite. Examples of cyclic presentations of the trivial group are of interest in connection with Andrews-Curtis conjecture [1] and have been researched in [15],[24] and elsewhere. In contrast, papers such as [2], [6], [9], [16], [28], [33], [43] give conditions for a cyclically presented group to be infinite, and in [32] for it to be SQ-universal. The classification of finite cyclically presented groups within certain families is a problem addressed in, for example, [14], [23], [42], [45].

In this paper we consider the "freeness" properties of largeness, SQ-universality, and the existence of free subgroups of rank 2. We investigate these properties both for arbitrary cyclically presented groups $G_{n}(w)$ and for the following family of groups, introduced and studied by Prishchepov in [33] and investigated further in [13],[40]. Let $n, r, s \geq 1,1 \leq k \leq n, 0 \leq q \leq n-1$ and define the Prischepov group to be

$$
\begin{aligned}
P(r, n, k, s, q) & =G_{n}\left(\left(x_{0} x_{q} \ldots x_{q(r-1)}\right)\left(x_{(k-1)} x_{(k-1)+q} \ldots x_{(k-1)+q(s-1)}\right)^{-1}\right) \\
& =\left\langle x_{0}, \ldots, x_{n-1} \mid x_{i} x_{i+q} \ldots x_{i+q(r-1)}=x_{i+(k-1)} x_{i+(k-1)+q} \ldots x_{i+(k-1)+q(s-1)}(0 \leq i<n)\right\rangle .
\end{aligned}
$$

This family contains various other families of cyclically presented groups that have been considered in the literature, starting with Conway's Fibonacci groups $F(2, n)=P(2, n, 3,1,1)$ of [14]. When $s=1$ 
the Prischepov groups coincide with Campbell and Robertson's Fibonacci-type groups $R(r, n, k, h)=$ $P(r, n,(r-1) h+k+1,1, h)$ of [5], which in turn contain the Fibonacci groups $F(r, n)=P(r, n, r+1,1,1)$ of [6], the generalized Fibonacci groups $F(r, n, k)=P(r, n, r+k, 1,1)$ of [7]; the Sieradski groups $S(2, n)=P(2, n, 2,1,2)$ of [39]; the Gilbert-Howie groups $H(n, t)=P(2, n, 2,1, t)$ of [23]; the socalled Cavicchioli-Hegenbarth-Repovš groups $G_{n}(m, k)=P(2, n, k+1,1, m)$ which were introduced independently in [11] and [27]. For $s \geq 1$ we have the groups $F(r, n, k, s)=P(r, n, r+k, s, 1)$ of [8] which contain the groups $H(r, n, s)=P(r, n, r+1, s, 1)$ of [6]; and we have the generalized Sieradski groups $S(r, n)=P(r, n, 2, r-1,2)(r \geq 2)$ of [10]. We remark that there would be certain advantages in defining $P(r, n, k, s, q)$ to be the group $G_{n}\left(\left(x_{0} x_{q} \ldots x_{q(r-1)}\right)\left(x_{k} x_{k+q} \ldots x_{k+q(s-1)}\right)^{-1}\right)$ (and this was done in [41]) but in order to maintain consistency with [33], and also with [40], we use Prischepov's original definition.

We start by giving some definitions and background material in Section 2. In Section 3 we use free products, epimorphic images, amalgamated free products, and a Freiheitssatz to obtain conditions under which a cyclically presented group $G_{n}(w)$ is large, SQ-universal, or contains a free subgroup of rank 2. In corollaries we apply these results to the Prishchepov groups. In Section 4 we obtain other basic properties of these groups. In Section 5 we study $P(r, n, k, s, q)$ in greater depth by finding new large epimorphic images and by applying Freiheitssatz results of Shwartz [35].

Except for three groups the finite groups $H(n, t)$ were classified in [23],[30]; one of the outstanding cases was proved infinite in [12]. Except for the two remaining unresolved groups in the family $H(n, t)$ the finite groups $G_{n}(m, k)$ were classified in [45],[46]. In Section 6 we obtain a new proof of this (almost complete) classification; this proof avoids the algebraic number theory results of [30],[45] that were required in the first proof.

\section{Preliminaries}

A group $G$ is large if it has a finite index subgroup that maps onto the free group of rank 2; $G$ is $S Q$-universal if every countable group can be embedded in a quotient group of $G$. Any large group is SQ-universal and hence contains a free subgroup of rank 2. Not every SQ-universal group is large however, even within the class of cyclically presented groups: the Higman group $G_{4}\left(x_{0} x_{1} x_{0}^{-2} x_{1}^{-1}\right)$ [25], which was proved to be SQ-universal in [34], has no proper subgroup of finite index and so cannot map onto the free group of rank 2 . As is well known, not every group containing a free subgroup of rank 2 is SQ-universal and so we can consider three distinct levels of 'freeness': largeness, SQ-universality, and the existence of free subgroups of rank 2. Each of these properties is preserved when taking finite extensions or finite index subgroups; also, a group that maps onto a group with one of these freeness properties also satisfies that property.

A free product $H * K$ (where $H, K$ are non-trivial) is large if and only if either $H$ or $K$ is large, or $H, K$ have non-trivial finite homomorphic images $\bar{H}, \bar{K}$, not both of order 2 ([31, Theorem 3.7]). An amalgamated free product $H *_{L} K$ in which $[H: L] \geq 2,[K: L] \geq 2$ and $[H: L]+[K: L] \geq 5$ contains a free subgroup of rank 2 (this is well known but see, for example, [4, Lemma 1]); if additionally $L$ is finite then the amalgamated free product is SQ-universal [29].

The automorphism $\theta$ of the introduction induces an action of the cyclic group $T=\left\langle t \mid t^{n}\right\rangle$ of order $n$ on the presentation $G_{n}(w)$. Specifically, $t^{-1} x_{i} t=x_{i+1}(0 \leq i \leq n-1)$ and therefore $t^{-i} x_{0} t^{i}=$ $x_{i}$. Writing $x=x_{0}$ we see that the split extension of $G_{n}(w)$ by $T$ has a presentation $E_{n}(W)=$ $\left\langle x, t \mid t^{n}=W(x, t)=1\right\rangle$ where $W(x, t)=x^{\alpha_{1}} t^{\beta_{1}} \ldots x^{\alpha_{\ell}} t^{\beta_{\ell}}$ (for some $\ell \geq 1,1 \leq \beta_{i} \leq n-1, \alpha_{i} \in \mathbb{Z} \backslash\{0\}$ $(1 \leq i \leq \ell))$ is a rewrite of $w=w\left(x_{0}, \ldots, x_{n-1}\right)$. We remark that $t$ has order $n$ in $E_{n}(W)$ and that if 
$w$ is an $m$ th power then $W(x, t)$ is also an $m$ th power.

In the case of a Prischepov group $P(r, n, k, s, q)$ the relator

$$
\left(x_{0} x_{q} \ldots x_{q(r-1)}\right)\left(x_{(k-1)} x_{(k-1)+q} \ldots x_{(k-1)+q(s-1)}\right)^{-1}
$$

rewrites to $\left(x t^{-q}\right)^{r-1} x t^{-B} x^{-1}\left(x t^{-q}\right)^{1-s} t^{A}$, where $A=(k-1), B=(k-1)-q(r-s)$. Setting $y=t^{q} x^{-1}$ and eliminating $x$ this becomes $y^{-r} t^{-B} y^{s} t^{A}$ and so the split extension of $P(r, n, k, s, q)$ by $T$ has a presentation

$$
M(r, n, k, s, q)=\left\langle y, t \mid t^{n}=1, y^{s} t^{A}=t^{B} y^{r}\right\rangle
$$

By the above comments $P(r, n, k, s, q)$ is large, SQ-universal, or contains a free subgroup of rank 2 if and only if $M(r, n, k, s, q)$ is large, SQ-universal, or contains a free subgroup of rank 2 , respectively.

\section{$3 \quad$ Free subgroups in cyclically presented groups}

\subsection{Free product of cyclically presented groups}

The following theorem, formalizing a statement made in the introduction of [15], gives conditions under which a cyclically presented group $G_{n}(w)$ can be expressed as a free product; its corollary gives conditions for it to be large.

Theorem 3.1 Let $w$ be a word in $x_{0}, \ldots, x_{n-1}$ involving only the $m$ letters $x_{\lambda_{0}}, \ldots, x_{\lambda_{m-1}}\left(0 \leq \lambda_{1}<\right.$ $\left.\lambda_{2}<\ldots<\lambda_{m-1} \leq n-1\right)$ so that $w=v\left(x_{\lambda_{0}}, \ldots, x_{\lambda_{m-1}}\right)$. Let $\Delta=\left(\lambda_{0}, \ldots, \lambda_{m-1}, n\right), N=n / \Delta$ and $\mu_{0}=\lambda_{0} / \Delta, \ldots, \mu_{m-1}=\lambda_{m-1} / \Delta$. Then $G_{n}(w)=G_{n}\left(v\left(x_{\lambda_{0}}, \ldots, x_{\lambda_{m-1}}\right)\right)$ is isomorphic to the free product of $\Delta$ copies of $G_{N}\left(v\left(x_{\mu_{0}}, \ldots, x_{\mu_{m-1}}\right)\right)$.

\section{Proof}

The group $G_{n}\left(v\left(x_{\lambda_{0}}, \ldots, x_{\lambda_{m-1}}\right)\right)$ has a presentation $\langle X \mid R\rangle$ where

$$
\begin{aligned}
X & =\left\{x_{i} \mid 0 \leq i \leq n-1\right\}, \\
R & =\left\{v\left(x_{\lambda_{0}+i}, x_{\lambda_{1}+i}, \ldots, x_{\lambda_{m-1}+i}\right) \mid 0 \leq i \leq n-1, \text { subscripts mod } n\right\} .
\end{aligned}
$$

For each $0 \leq \alpha \leq \Delta-1$ set

$$
\begin{aligned}
X_{\alpha}= & \left\{x_{i} \mid i \equiv \alpha \bmod \Delta, 0 \leq i \leq n-1\right\} \\
= & \left\{x_{\alpha}, x_{\alpha+\Delta}, \ldots, x_{\alpha+(N-1) \Delta}\right\} \\
R_{\alpha}= & \left\{v\left(x_{\lambda_{0}+i}, x_{\lambda_{1}+i}, \ldots, x_{\lambda_{m-1}+i}\right) \mid i \equiv \alpha \bmod \Delta, 0 \leq i \leq n-1\right\} \\
= & \left\{v\left(x_{\lambda_{0}+\alpha}, x_{\lambda_{1}+\alpha}, \ldots, x_{\lambda_{m-1}+\alpha}\right), v\left(x_{\lambda_{0}+\alpha+\Delta}, x_{\lambda_{1}+\alpha+\Delta}, \ldots, x_{\lambda_{m-1}+\alpha+\Delta}\right), \ldots,\right. \\
& \left.v\left(x_{\lambda_{0}+\alpha+(N-1) \Delta}, x_{\lambda_{1}+\alpha+(N-1) \Delta}, \ldots, x_{\lambda_{m-1}+\alpha+(N-1) \Delta}\right)\right\} .
\end{aligned}
$$

Then $R_{\alpha}$ is a set of words involving only elements of $X_{\alpha}$ and the $X_{\alpha}$ form a partition of $X$ and the $R_{\alpha}$ form a partition of $R$. Hence

$$
\langle X \mid R\rangle \cong\left\langle X_{0} \mid R_{0}\right\rangle * \ldots *\left\langle X_{N-1} \mid R_{N-1}\right\rangle .
$$

Fix a value of $\alpha(0 \leq \alpha \leq N-1)$ and set $y_{0}=x_{\alpha}, y_{1}=x_{\alpha+\Delta}, \ldots, y_{(N-1)}=x_{\alpha+(N-1) \Delta}$. Then $X_{\alpha}=\left\{y_{0}, \ldots, y_{N-1}\right\}$ and $x_{\lambda_{0}+\alpha}=y_{\mu_{0}}, x_{\lambda_{1}+\alpha}=y_{\mu_{1}}, \ldots, x_{\lambda_{m-1}+\alpha}=y_{\mu_{m-1}}$ so $R_{\alpha}$ is the set

$$
\left\{v\left(y_{\mu_{0}}, y_{\mu_{1}}, \ldots, y_{\mu_{m-1}}\right), v\left(y_{\mu_{0}+1}, y_{\mu_{1}+1}, \ldots, y_{\mu_{m-1}+1}\right), \ldots, v\left(y_{\mu_{0}+(N-1)}, y_{\mu_{1}+(N-1)}, \ldots, y_{\mu_{m-1}+(N-1)}\right\}\right. \text {. }
$$

Thus $\left\langle X_{\alpha} \mid R_{\alpha}\right\rangle \cong G_{N}\left(v\left(y_{\mu_{0}}, y_{\mu_{1}}, \ldots, y_{\mu_{m-1}}\right)\right)$ which (by relabeling) is $G_{N}\left(v\left(x_{\mu_{0}}, x_{\mu_{1}}, \ldots, x_{\mu_{m-1}}\right)\right)$ and the result follows. 
Corollary 3.2 With the above notation let $G=G_{n}\left(v\left(x_{\lambda_{0}}, \ldots, x_{\lambda_{m-1}}\right)\right), H=G_{N}\left(v\left(x_{\mu_{0}}, \ldots, x_{\mu_{m-1}}\right)\right)$ and suppose $\Delta \geq 2, H \neq 1$. Then $G$ is large unless $\Delta=2$ and $H \cong \mathbb{Z}_{2}$, in which case $G \cong D_{\infty}$.

Now $|r-s|$ divides the determinant of the relation matrix of $P(r, n, k, s, q)$ and so it divides $|P(r, n, k, s, q)|$. Using this and applying Theorem 3.1 and Corollary 3.2 to Prischepov groups we have

Corollary 3.3 Let $\Delta=(n, k-1, q)$ when $r+s \geq 3$ and let $\Delta=(n, k-1)$ when $r=s=1$. Then $P=P(r, n, k, s, q)$ is isomorphic to the free product of $\Delta$ copies of $H=P(r, N, K, s, Q)$ where $N=n / \Delta, Q=q / \Delta, K=(k-1) / \Delta+1$. If $H \neq 1, \Delta \geq 2$ then $P$ is large unless $H \cong \mathbb{Z}_{2}$ and $\Delta=2$, in which case $P \cong D_{\infty}$. In particular, if $\Delta \geq 2$ and $|r-s| \neq 1$ then $P$ is large unless $\Delta=2$ and $|r-s|=2$.

In particular we recover a result about the groups $R(r, n, k, h)$.

Corollary 3.4 ([5, Theorem 4]) The group $R(r, n, k, h)$ is isomorphic to the free product of $(n, k, h)$ copies of $R(r, N, K, H)$ where $N=n /(n, k, h), K=k /(n, k, h), H=h /(n, k, h)$.

In particular, if $(n, k, h)>1$ then $R(r, n, k, h)$ is large unless $R(r, N, K, H)=1$ or $((n, k, h)=2$, $r \leq 3$ and $\left.R(r, N, K, H) \cong \mathbb{Z}_{2}\right)$. This corollary in turn contains a result about the groups $G_{n}(m, k)$.

Corollary 3.5 ([2, Lemma 1.2]) The group $G_{n}(m, k)$ is isomorphic to the free product of $(n, m, k)$ copies of $G_{N}(M, K)$ where $N=n /(n, m, k), M=m /(n, m, k), K=k /(n, m, k)$.

In particular, if $(n, m, k)>1$ then $G_{n}(m, k)$ is large unless $G_{N}(M, K)=1$ or $((n, m, k)=2$ and $\left.G_{N}(M, K) \cong \mathbb{Z}_{2}\right)$.

\subsection{Epimorphic images}

If a group $G$ maps homomorphically onto a large group, or onto a group that contains a free subgroup of rank 2 then $G$ is large, or contains a free subgroup of rank 2, respectively. Our method of proof in this section is to find suitable epimorphic images of $E_{n}(W)$.

It was determined in [19] when the group $\left\langle x, t \mid t^{n}=W(x, t)^{m}=1\right\rangle(m \geq 2)$ contains a free subgroup of rank 2 or is infinite and soluble. (Actually, it also gives conditions under which the group contains a Ree-Mendelsohn pair - see [19] for the definition - or is infinite and soluble.) Combining that theorem with [3] we can prove the following related result.

Theorem 3.6 Let $E_{n}(W)=\left\langle x, t \mid t^{n}=V(x, t)^{m}=1\right\rangle$ where $n, m \geq 2$ and $V(x, t)=x^{\alpha_{1}} t^{\beta_{1}} \ldots x^{\alpha_{\ell}} t^{\beta_{\ell}}$, $\ell \geq 1,1 \leq \beta_{i} \leq n-1, \alpha_{i} \in \mathbb{Z} \backslash\{0\}(1 \leq i \leq \ell)$.

(a) If $n+m \geq 5$ then $E_{n}(W)$ is large;

(b) if $n=m=2$ then $E_{n}(W)$ contains a free subgroup of rank 2 unless $\ell=1$ and $\alpha_{1} \leq 2$, in which case $E_{n}(W)$ is infinite and soluble.

\section{Proof}

If $n+m \geq 5$ then choose $k \in \mathbb{N}$ with $k>\max \left\{6,\left|\alpha_{1}\right|, \ldots,\left|\alpha_{\ell}\right|\right\}$. Then $E_{n}(W)$ maps onto the group $\left\langle x, t \mid x^{k}=t^{n}=V(x, t)^{m}=1\right\rangle$ which is large by [3] since $1 / k+1 / n+1 / m<1$. If $n=m=2$ then the result was proved in [19, Theorem 4] (see also [20, Theorem 8] or [21, Theorem 7.3.3.1]). 
Theorem 3.7 Let $E_{n}(W)=\left\langle x, t \mid t^{n}=W(x, t)=1\right\rangle$ where $W(x, t)=x^{\alpha_{1}} t^{\beta_{1}} \ldots x^{\alpha_{\ell}} t^{\beta_{\ell}}, \ell \geq 1,1 \leq$ $\beta_{i} \leq n-1, \alpha_{i} \in \mathbb{Z} \backslash\{0\}(1 \leq i \leq \ell)$.

(a) If $\left(\beta_{1}, \ldots, \beta_{\ell}, n\right) \geq 2$ and $\left|\sum_{i=1}^{\ell} \alpha_{i}\right| \neq 1$ then $E_{n}(W)$ is large except possibly when $\left(\beta_{1}, \ldots, \beta_{\ell}, n\right)=$ 2 and $\left|\sum_{i=1}^{\ell} \alpha_{i}\right|=2$, in which case $E_{n}(W)$ is infinite.

(b) If $\left(n, \sum_{i=1}^{\ell} \beta_{i}\right) \geq 2$ and $\left(\alpha_{1}, \ldots, \alpha_{\ell}\right) \geq 2$ then $E_{n}(W)$ is large except possibly when $\left(n, \sum_{i=1}^{\ell} \beta_{i}\right)=$ 2 and $\left(\alpha_{1}, \ldots, \alpha_{\ell}\right)=2$, in which case $E_{n}(W)$ is infinite.

\section{Proof}

For (a) observe that the group $E_{n}(W)$ maps onto $\left\langle x, t \mid t^{\left(\beta_{1}, \ldots, \beta_{\ell}, n\right)}=x^{\left|\sum_{i=1}^{\ell} \alpha_{i}\right|}=1\right\rangle \cong \mathbb{Z}_{\left(\beta_{1}, \ldots, \beta_{\ell}, n\right)} *$ $\mathbb{Z}_{\left|\sum_{i=1}^{\ell} \alpha_{i}\right|}$ and for (b) that it maps onto $\left\langle x, t \mid t^{\left(n, \sum_{i=1}^{\ell} \beta_{i}\right)}=x^{\left(\alpha_{1}, \ldots, \alpha_{\ell}\right)}=1\right\rangle \cong \mathbb{Z}_{\left(n, \sum_{i=1}^{\ell} \beta_{i}\right)} * \mathbb{Z}_{\left(\alpha_{1}, \ldots, \alpha_{\ell}\right)}$.

Corollary 3.8 (a) If $(n, A, B) \geq 2$ and $|r-s| \neq 1$ then $M(r, n, k, s, q)$ is large except possibly when $(n, A, B)=2$ and $|r-s|=2$, in which case it is infinite.

(b) If $(n, A-B) \geq 2$ and $(r, s) \geq 2$ then $M(r, n, k, s, q)$ is large except possibly when $(n, A-B)=2$ and $(r, s)=2$, in which case it is infinite.

As an immediate corollary we get

Corollary 3.9 ([44, Theorems 1 and 2]) If $(r, n)>1$ then $F(r+1, n, 0)$ is infinite.

\subsection{Amalgamated free products and the Freiheitssatz}

The following theorem uses the fact that the group $E_{n}(W)=\left\langle x, t \mid t^{n}=W(x, t)=1\right\rangle$ can sometimes be expressed as an amalgamated free product, possibly with the amalgamation over a finite group, to prove SQ-universality of $E_{n}(W)$ or the existence of a free subgroup of rank 2. Since the split extension of any cyclically presented group $G_{n}(w)$ is of the form $E_{n}(W)$ the theorem can be used to prove SQ-universality of $G_{n}(w)$ or the existence of a free subgroup of rank 2.

Theorem 3.10 Let $E_{n}(W)=\left\langle x, t \mid t^{n}=W(x, t)=1\right\rangle$ where $n \geq 2$ and $W(x, t)=x^{\alpha_{1}} t^{\beta_{1}} \ldots x^{\alpha_{\ell}} t^{\beta_{\ell}}$, $\ell \geq 1,1 \leq \beta_{i} \leq n-1, \alpha_{i} \in \mathbb{Z} \backslash\{0\}(1 \leq i \leq \ell)$, and suppose $x$ has infinite order and $t$ has order $n$ in $E_{n}(W)$.

(a) If $\left(\alpha_{1}, \ldots, \alpha_{\ell}\right) \geq 2, n \geq 3$ then $E_{n}(W)$ contains a free subgroup of rank 2.

(b) If $\left(\beta_{1}, \ldots, \beta_{\ell}, n\right) \geq 2$ then $E_{n}(W)$ is SQ-universal.

In particular, if $n \geq 3$ and $E_{n}(W)$ does not contain a free subgroup of rank 2 then $\left(\alpha_{1}, \ldots, \alpha_{\ell}\right)=1$ and $\left(\beta_{1}, \ldots, \beta_{\ell}, n\right)=1$.

\section{Proof}

(a) Let $a=\left(\alpha_{1}, \ldots, \alpha_{\ell}\right), \gamma_{i}=\alpha_{i} / a(1 \leq i \leq \ell)$. Then $E_{n}(W) \cong H *_{L} K$ where

$$
H=\left\langle x^{a}, t \mid t^{n}=\left(x^{a}\right)^{\gamma_{1}} t^{\beta_{1}} \ldots\left(x^{a}\right)^{\gamma_{\ell}} t^{\beta_{\ell}}=1\right\rangle,
$$

$K=\langle x \mid\rangle, L=\left\langle x^{a} \mid\right\rangle$. Now $[K: L]=a \geq 2$. If $[H: L]=1$ or 2 then $H \cong \mathbb{Z}$ or $D_{\infty}$. But $t$ has order $n \geq 3$ in $H$ so $H \neq D_{\infty}$. Further, $H \neq \mathbb{Z}$ since $t$ has order $n$. Thus $[H: L] \geq 3$. 
(b) Let $b=\left(\beta_{1}, \ldots, \beta_{\ell}, n\right), \delta_{i}=\beta_{i} / b(1 \leq i \leq \ell), N=n / b$. Then $E_{n}(W) \cong H *_{L} K$ where $H=\left\langle x, t^{b} \mid\left(t^{b}\right)^{N}=x^{\alpha_{1}}\left(t^{b}\right)^{\delta_{1}} \ldots x^{\alpha_{\ell}}\left(t^{b}\right)^{\delta_{\ell}}=1\right\rangle, K=\left\langle t \mid t^{n}\right\rangle, L=\left\langle t^{b} \mid\left(t^{b}\right)^{N}\right\rangle$. Now $[K: L]=b \geq 2$ and $L$ has infinite index in $H$ since $L$ is finite and $H$ is infinite.

If $E_{n}(W)$ arises as a split extension of the group $G_{n}(w)$, as explained in Section 2, then $t$ has order $n$ in $E_{n}(W) ; x$ will not always have infinite order of course. When $\sum_{i=1}^{\ell} \alpha_{i}=0$, however, there is an epimorphism $E \rightarrow \mathbb{Z}$ given by $t \mapsto 0, x \mapsto 1 \in \mathbb{Z}$ and so $x$ has infinite order in $E_{n}(W)$.

We now consider the hypothesis " $x$ has infinite order and $t$ has order $n$ in $E_{n}(W)$ " in more detail. A one-relator product $G=(H * K) /<<R>>$ (where $<<R>>$ denotes the normal closure of $R$ in $H * K$ ) is said to satisfy the Freiheitssatz if the natural homomorphisms $H \rightarrow G, K \rightarrow G$ are both embeddings. The Freiheitssatz for one-relator products has been considered in many papers - see [17],[18],[26],[38] and the references therein. Setting $H=\langle x \mid\rangle \cong \mathbb{Z}, K=\left\langle t \mid t^{n}\right\rangle \cong \mathbb{Z}_{n}$, $R=W(x, t)$ we see that $E_{n}(W)=(H * K) /<<R>>$. Clearly the Freiheitssatz holds here if and only if $x$ has infinite order and $t$ has order $n$ in $E_{n}(W)$. Thus we can re-express Theorem 3.10 as

Theorem 3.10' Let $E_{n}(W)=(H * K) /<<R>>$ where $H=\langle x \mid\rangle \cong \mathbb{Z}, K=\left\langle t \mid t^{n}\right\rangle \cong \mathbb{Z}_{n}$, $R=W(x, t)$ where $n \geq 2$ and $W(x, t)=x^{\alpha_{1}} t^{\beta_{1}} \ldots x^{\alpha_{\ell}} t^{\beta_{\ell}}, \ell \geq 1,1 \leq \beta_{i} \leq n-1, \alpha_{i} \in \mathbb{Z} \backslash\{0\}$ $(1 \leq i \leq \ell)$, and suppose that the Freiheitssatz holds.

(a) If $\left(\alpha_{1}, \ldots, \alpha_{\ell}\right) \geq 2, n \geq 3$ then $E_{n}(W)$ contains a free subgroup of rank 2.

(b) If $\left(\beta_{1}, \ldots, \beta_{\ell}, n\right) \geq 2$ then $E_{n}(W)$ is SQ-universal.

In particular, if $n \geq 3$ and $E_{n}(W)$ does not contain a free subgroup of rank 2 then $\left(\alpha_{1}, \ldots, \alpha_{\ell}\right)=1$ and $\left(\beta_{1}, \ldots, \beta_{\ell}, n\right)=1$.

Applying this to Prischepov groups we have

Corollary 3.11 Let $M=M(r, n, k, s, q)$ where $n \geq 2$. Then $M$ is the one-relator product $(H * K) /<<$ $R>>$ where $\{H, K\}=\left\{\langle x \mid\rangle,\left\langle t \mid t^{n}\right\rangle\right\}, R=y^{s} t^{A} y^{-r} t^{-B}$ where $A=(k-1), B=(k-1)-q(r-s)$. Suppose that the Freiheitssatz holds.

(a) If $(r, s) \geq 2, n \geq 3$ then $M$ contains a free subgroup of rank 2 .

(b If $(A, B, n) \geq 2$ then $M$ is SQ-universal.

In particular, if $n \geq 3$ and $M$ does not contain a free subgroup of rank 2 then $(r, s)=1$ and $(A, B, n)=$ 1.

(We remark that alternative forms of the Freiheitssatz for cyclically presented groups and their extensions have been considered in [16],[28].)

\section{Basic properties of Prishchepov groups}

We first note some isomorphisms amongst the groups $P(r, n, k, s, q)$.

Lemma 4.1 $P(r, n, k, s, q) \cong P\left(r^{\prime}, n, k^{\prime}, s^{\prime}, q\right)$, where $r^{\prime}=s, s^{\prime}=r, k^{\prime}=n-k+2$. 


\section{Proof}

Let $r^{\prime}, s^{\prime}, k^{\prime}$ be as stated and for each $0 \leq i \leq n-1$, set $j=i+(k-1) \bmod n$. Then the relators of $P(r, n, k, s, q)$, namely $\left(x_{i} x_{i+q} \ldots x_{i+q(r-1)}\right)\left(x_{i+(k-1)} x_{i+(k-1)+q} \ldots x_{i+(k-1)+q(s-1)}\right)^{-1}$ become $\left(x_{j+\left(k^{\prime}-1\right)} x_{j+\left(k^{\prime}-1\right)+q} \ldots x_{j+\left(k^{\prime}-1\right)+q(r-1)}\right)\left(x_{j} x_{j+q} \ldots x_{j+q(s-1)}\right)^{-1}$. Inverting these we get the relators $\left(x_{j} x_{j+q} \ldots x_{j+q(s-1)}\right)\left(x_{j+\left(k^{\prime}-1\right)} x_{j+\left(k^{\prime}-1\right)+q} \ldots x_{j+\left(k^{\prime}-1\right)+q(r-1)}\right)^{-1}$ which are the relators of $P\left(r^{\prime}, n, k^{\prime}, s^{\prime}, q\right)$.

Thus the roles of $r, s$ may be interchanged. For $P(r, n, k, s, q)$ we have $A=(k-1), B=(k-$ 1) $-q(r-s)$; the corresponding values for $P\left(r^{\prime}, n, k^{\prime}, s^{\prime}, q\right)$ are $A^{\prime}=\left(k^{\prime}-1\right) \equiv-A \bmod n, B^{\prime}=$ $\left(k^{\prime}-1\right)-q\left(r^{\prime}-s^{\prime}\right) \equiv-B \bmod n-$ that is, $A$ and $B$ are negated $(\bmod n)$.

Lemma $4.2 \quad$ (i) $P(r, n, k, s, q) \cong P(r, n, k-q(r-s), s, n-q)$;

(ii) $P(r, n, k, s, q) \cong P(s, n, k-q(r-s), r, q)$.

\section{Proof}

(i) Setting $y_{i}=x_{i}^{-1}(0 \leq i \leq n-1$, subscripts $\bmod n)$ the relators

$$
\left(x_{i} x_{i+q} \ldots x_{i+q(r-2)} x_{i+q(r-1)}\right)\left(x_{i+(k-1)} x_{i+(k-1)+q} \ldots x_{i+(k-1)+q(s-2)} x_{i+(k-1)+q(s-1)}\right)^{-1}
$$

of $P(r, n, k, s, q)$ become

$$
\left(y_{i}^{-1} y_{i+q}^{-1} \ldots y_{i+q(r-2)}^{-1} y_{i+q(r-1)}^{-1}\right)\left(y_{i+(k-1)}^{-1} y_{i+(k-1)+q}^{-1} \ldots y_{i+(k-1)+q(s-2)}^{-1} y_{i+(k-1)+q(s-1)}^{-1}\right)^{-1}
$$

which is a cyclic permutation of

$$
\left(y_{i+(k-1)+q(s-1)} y_{i+(k-1)+q(s-2)} \ldots y_{i+(k-1)+q} y_{i+(k-1)}\right)\left(y_{i+q(r-1)} y_{i+q(r-2)} \ldots y_{i+q} y_{i}\right)^{-1} .
$$

Inverting gives

$$
\left(y_{i+q(r-1)} y_{i+q(r-2)} \ldots y_{i+q} y_{i}\right)\left(y_{i+(k-1)+q(s-1)} y_{i+(k-1)+q(s-2)} \ldots y_{i+(k-1)+q} y_{i+(k-1)}\right)^{-1}
$$

and then setting $j=i+q(r-1) \bmod n$ (for each $0 \leq i \leq n-1)$ these become

$$
\begin{aligned}
& \left(y_{j} y_{j+(n-q)} \ldots y_{j+(r-2)(n-q)} y_{j+(r-1)(n-q)}\right) \\
& \quad\left(y_{j+(k-1)-q(r-s)} y_{j+(k-1)-q(r-s)+(n-q)} \ldots y_{j+(k-1)-q(r-s)+(s-2)(n-q)} y_{j+(k-1)-q(r-s)+(s-1)(n-q)}\right)^{-1}
\end{aligned}
$$

which are the relators of $P(r, n, k-q(r-s), s, n-q)$.

(ii) Setting $z_{i}=y_{-i}(0 \leq i \leq n-1$, subscripts $\bmod n)$ in (1) we get

$$
\left(z_{-i-(k-1)-q(s-1)} z_{-i-(k-1)-q(s-2)} \ldots z_{-i-(k-1)-q} z_{-i-(k-1)}\right)\left(z_{-i-q(r-1)} z_{-i-q(r-2)} \ldots z_{-i-q} z_{-i}\right)^{-1} .
$$

Letting $j=-i-(k-1)-q(s-1) \bmod n$ (for each $0 \leq i \leq n-1)$ these become

$$
\begin{aligned}
& \left(z_{j} z_{j+q} \ldots z_{j+q(s-2)} z_{j+q(s-1)}\right) \\
& \quad\left(z_{j+(k-1)+q(s-r)} z_{j+(k-1)+q(s-r)+q} \ldots z_{j+(k-1)+q(s-r)+q(r-2)} z_{j+(k-1)+q(s-r)+q(r-1)}\right)^{-1} .
\end{aligned}
$$

These are the relators of $P(s, n, k-q(r-s), r, q)$ so the proof is complete.

For $P(r, n, k, s, q)$ we have $A=(k-1), B=(k-1)-q(r-s)$; the corresponding values for the isomorphic copy of $P(r, n, k, s, q)$ (in either (i) or (ii)) are $A^{\prime}=B, B^{\prime}=A$ so the roles of $A, B$ may also be interchanged. Thus while part (ii) interchanges the roles of $r, s$ we now have a different effect on $A, B$ than that obtained when we use Lemma 4.1. 
Corollary 4.3 ([2, Lemma 1.1(3)]) $G_{n}(m, k) \cong G_{n}(n-m, n+(k-m))$.

Applying the technique used in [5, Lemma 2] more generally we have

Theorem 4.4 Let $(\alpha, n)=1$. Then $G_{n}\left(w\left(x_{0}, x_{1}, \ldots, x_{n-1}\right)\right) \cong G_{n}\left(w\left(x_{0}, x_{\alpha}, \ldots, x_{\alpha(n-1)}\right)\right)$. In particular if $(q, n)=1$ then $P(r, n, k, s, q) \cong P(r, n,(k-1) Q+1, s, 1)$ where $q Q \equiv 1 \bmod n$.

\section{Proof}

Let $a$ satisfy $a \alpha \cong 1 \bmod n$ and for each $0 \leq j \leq n-1$ set $i=a j \bmod n$, so $j \equiv \alpha i \bmod n$ and define $y_{i}=x_{\alpha i}(0 \leq i \leq n-1$, subscripts $\bmod n)$. Then the set of generators of $G_{n}\left(w\left(x_{0}, x_{\alpha}, \ldots, x_{\alpha(n-1)}\right)\right)$ $\left\{x_{0}, x_{1}, \ldots, x_{n-1}\right\}=\left\{y_{0}, y_{1}, \ldots, y_{n-1}\right\}$ and the set of relators

$$
\left\{w\left(x_{j}, x_{j+\alpha}, \ldots, x_{j+(n-1) \alpha}\right) \mid 0 \leq j \leq n-1\right\}=\left\{w\left(y_{i}, y_{i+1}, \ldots, y_{i+(n-1)}\right) \mid 0 \leq i \leq n-1\right\}
$$

and the result follows.

As a corollary we of course recover [5, Lemma 2] which states that $R(r, n, k, h) \cong R(r, n, \alpha k, \alpha h)$ for any $(\alpha, n)=1$. This in turn implies the following, which we record for later use.

Corollary $4.5\left(\left[2\right.\right.$, Lemma 1.3]) (i) If $(n, k)=1$ then $G_{n}(m, k) \cong G_{n}(t, 1)=H(n, t)$ where $t k=m \bmod n$.

(ii) If $(n, k-m)=1$ then $G_{n}(m, k) \cong G_{n}(t, 1)=H(n, t)$ where $t(k-m)=n-m \bmod n$.

Let $P=P(r, n, k, s, q)$. If $A \equiv 0 \bmod n$ then $k \equiv 1 \bmod n$ so $P=P(r, n, 1, s, q)$; if $B \equiv 0 \bmod n$ then $k-q(r-s) \equiv 1 \bmod n$ and using the equivalent presentation $P(s, n, k-q(r-s), r, q)$ (of Lemma 4.2(ii)) we see that $P \cong P(s, n, 1, r, q)$. Furthermore, a direct consideration of the cyclic presentation shows that $P(r, n, 1, s, q)=P(|r-s|+1, n, 1,1, q)$. We can classify when these groups are large:

Theorem 4.6 Let $P=P(r, n, 1,1, q)$ with $r \geq 1$ and let $d=(n,(r-1) q)$.

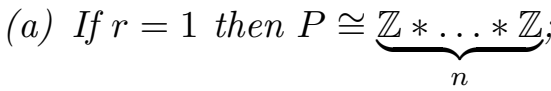

(b) if $r=2$ then $P=1$;

(c) if $d=1$ then $P \cong \mathbb{Z}_{r-1}$;

(d) if $r=3$ and $d=2$ then $P \cong \begin{cases}D_{\infty} & \text { if }(n, q)=2 \text { and } n=2 \bmod 4, \\ \mathbb{Z} & \text { if }(n, q)=1 ;\end{cases}$

(e) if $r \geq 4$ or $d \geq 3$ then $P$ is large.

\section{Proof}

The cases $r=1$ and $r=2$ (parts (a) and (b)) are immediate by considering the cyclic presentation.

Suppose $d=1$. Then $(n, q)=1$ so, by Theorem $4.4, P \cong P(r, n, 1,1,1)=G_{n}\left(x_{0} x_{1} \ldots x_{r-2}\right)$. Moreover $(n, r-1)=1$ so by [44, Theorem 3] $G_{n}\left(x_{0} x_{1} \ldots x_{r-2}\right) \cong \mathbb{Z}_{r-1}$, proving part (c). Suppose then that $r \geq 3$ and $d \geq 2$.

If $r \geq 4$ or $d \geq 3$ then Corollary 3.8 implies that $P$ is large so assume that $r=3$ and $d=$ 2 (i.e. $(n, 2 q)=2)$. Now Corollary 3.3 implies that $P$ is isomorphic to $(n, q)$ copies of $G=$ 
$P(3, N, 1,1, Q)$ where $N=n /(n, q), Q=q /(n, q)$, and since $(Q, N)=1$ Theorem 4.4 implies that $G \cong$ $P(3, N, 1,1,1)=G_{N}\left(x_{0} x_{1}\right)$. Eliminating generators $x_{N}, x_{N-1}$ shows that $G_{N}\left(x_{0} x_{1}\right) \cong G_{N-2}\left(x_{0} x_{1}\right)$ and it is clear that $G_{3}\left(x_{0} x_{1}\right) \cong \mathbb{Z}_{2}$ and $G_{2}\left(x_{0} x_{1}\right) \cong \mathbb{Z}$ so $G_{N}\left(x_{0} x_{1}\right) \cong \mathbb{Z}$ when $N$ is even and $G_{N}\left(x_{0} x_{1}\right) \cong \mathbb{Z}_{2}$ when $N$ is odd. If $(n, q)=2$ then $n \equiv 2 \bmod 4$, since $(n, 2 q)=2$, so $N$ is odd and hence $P \cong \mathbb{Z}_{2} * \mathbb{Z}_{2} \cong D_{\infty}$. If $(n, q)=1$ then $n=N$ so $P \cong \mathbb{Z}$ since $n$ is even.

\section{$5 \quad$ Free subgroups in Prishchepov groups}

\section{$5.1 \quad$ Largeness}

In this section we extend ideas that were first used in [9]. As in Section 3 we prove largeness by finding a large epimorphic image. When we consider (the split extension of) Prishchepov groups $P(r, n, k, s, q)$, rather than arbitrary cyclically presented groups, there is a new epimorphic image that we can use. Let $d=(n, A+B)$; then by killing $t^{d}$ we see that $M(r, n, k, s, q)$ maps onto

$$
N=\left\langle y, t \mid t^{d}=1,\left(y^{s} t^{A}\right)^{2}=y^{r+s}\right\rangle .
$$

(Note that $N=M(r, d,(r-s) q / 2+1, s, q)$.) The group $N$ in turn maps onto the generalized triangle group $\left\langle y, t \mid y^{r+s}=t^{d}=\left(y^{s} t^{A}\right)^{2}=1\right\rangle$.

Let $G(l, m, n)=\left\langle a, b \mid a^{l}=b^{m}=\left(a^{\alpha} b^{\beta}\right)^{n}=1\right\rangle$. In [19, Theorem 6] (see also [20, Theorem 2], [21, Theorem 7.3.2.2]) it was determined when $G(l, m, n)$ contains a free subgroup of rank 2 , is infinite and soluble, or is finite; independently in [9, Theorem 2.5] the finite groups $G(l, m, n)$ were classified. Refining these results slightly we can classify when $G(l, m, n)$ is large, infinite and soluble, or finite.

Theorem 5.1 Let $G=\left\langle a, b \mid a^{l}=b^{m}=\left(a^{\alpha} b^{\beta}\right)^{n}=1\right\rangle$ where $1 \leq \alpha \leq l-1,1 \leq \beta \leq m-1$ and let $\kappa=1 / l+1 / m+1 / n-1$.

(a) If $(\alpha, l)=1$ and $(\beta, m)=1$ then $G$ is large if $\kappa<0$, infinite and soluble if $\kappa=0$, finite if $\kappa>1$.

(b) If $(\alpha, l)>1$ or $(\beta, m)>1$ then $G$ is large unless either:

(i) $l=2, n=2$ and $(\beta, m)=2$; or

(ii) $m=2, n=2$ and $(\alpha, l)=2$;

in which case $G$ is infinite and soluble.

\section{Proof}

(a) If $(\alpha, l)=1$ and $(\beta, m)=1$ then we may assume $\alpha=\beta=1$, in which case $G$ is an ordinary triangle group and the result is well known. (b) If $\kappa<1$ then $G$ is large by [3, Theorem B]. If $\{l, m\}=$ $\{2,2\},\{2,3\},\{2,5\},\{3,3\}$, or $\{3,5\}$ then $(\alpha, l)=(\beta, m)=1$. Thus we only need to consider the cases $(\{l, m\}, n)=(\{2, k\}, 2)(k \geq 4),(\{3,4\}, 2),(\{3,6\}, 2),(\{2,4\}, 3),(\{2,6\}, 3),(\{2,4\}, 4),(\{4,4\}, 2)$ where $(\alpha, l)>1$ or $(\beta, m)>1$. If $(l, m, n)=(2, m, 2)$ then $G$ maps onto $\left\langle a, b \mid a^{2}=b^{(\beta, m)}=1\right\rangle$ which is large unless $(\beta, m)=2$ and in this case the cyclic subgroup $H=\left\langle b^{\beta} \mid b^{m}\right\rangle$ is normal in $G$ and $G / H \cong D_{\infty}$ so $G$ is infinite and soluble. Similarly, if $(l, m, n)=(l, 2,2)$ then $G$ is large unless $(\alpha, l)=2$ in which case $G$ is infinite and soluble. By passing to another generating pair if necessary we may assume $\alpha|l, \beta| m$ which means that for the remaining triples there are nine groups to consider. In each case we can use GAP [22] to find a subgroup (of index at most 6) that maps onto a free product of two cyclic groups 
(other than $\mathbb{Z}_{2} * \mathbb{Z}_{2}$ ) and hence $G$ is large.

We now classify the large 2-generator Prischepov groups.

Theorem 5.2 Let $M=M(r, 2, k, s, q), P=P(r, 2, k, s, q), A=(k-1), B=(k-1)-q(r-s)$. If $(r, s)=1$ let $\alpha, \beta \in \mathbb{Z}$ be such that $\alpha r+\beta s=1$ and set $g=\left|s^{2}-r^{2}\right|(\alpha, \beta)$. Then $M$ is large unless one of the following holds:

(a) $A, B$ are both even and either

(i) $|r-s|=2$ in which case $M \cong D_{\infty}$,

1. if $q$ is even then $P \cong D_{\infty}$;

2. if $q$ is odd then $P \cong \mathbb{Z}$;

(ii) $|r-s|=1$ in which case $M \cong \mathbb{Z}_{2}$ and $P=1$;

(b) $A, B$ are of opposite parity, in which case $M \cong \mathbb{Z}_{2|r-s|}, P \cong \mathbb{Z}_{|r-s|}$;

(c) A,B are both odd and one of the following holds:

(i) $(r, s)=2$, in which case $M$ and $P$ are infinite and soluble;

(ii) $r=s=1$, in which case $M \cong \mathbb{Z}_{2} \times \mathbb{Z}, P \cong \mathbb{Z}$;

(iii) $(r, s)=1$ and $r+s \geq 3$, in which case $M$ soluble and finite of order $2 g$,

1. if $q$ is even then $P \cong \mathbb{Z}_{g}$;

2. if $q$ is odd then $P$ is non-abelian and soluble of order $g$.

\section{Proof}

If $A, B$ are both even then $M \cong \mathbb{Z}_{2} * \mathbb{Z}_{|r-s|}$ which is large unless $|r-s|=2$ or 1 . If $|r-s|=2$ then $M \cong D_{\infty}$ and $P \cong D_{\infty}$ when $q$ is even and $P \cong \mathbb{Z}$ when $q$ is odd. If $|r-s|=1$ then $M \cong \mathbb{Z}_{2}$ so $P=1$. If $A, B$ are of opposite parity then $M \cong \mathbb{Z}_{2|r-s|}$ and hence $P \cong \mathbb{Z}_{|r-s|}$. Suppose then that $A, B$ are both odd.

Now $M=\left\langle y, t \mid t^{2}=1, y^{s} t=t y^{r}\right\rangle$ maps onto $\left\langle y, t \mid t^{2}=y^{(r, s)}=1\right\rangle$ which is large when $(r, s) \geq 3$ so assume $(r, s)=1$ or 2 . If $r=s=1$ then $M \cong \mathbb{Z}_{2} \times \mathbb{Z}$ and $P \cong \mathbb{Z}$ so assume $r+s \geq 3$. Let $G=\left\langle y, t \mid y^{r+s}=t^{2}=\left(y^{s} t\right)^{2}=1\right\rangle$. If $(r, s)=1$ then $G \cong D_{2(r+s)}$, which is soluble; if $(r, s)=2$ then Theorem 5.1 implies that $G$ is infinite and soluble. The cyclic subgroup $H=\left\langle\left(y^{s} t\right)^{2}\right\rangle$ is normal in $M$ and $M / H \cong G$, which is soluble, so $M$ is soluble. Since $M$ maps onto $G$ we have that $M$ is infinite when $(r, s)=2$. Assume then that $(r, s)=1$.

Suppose $q$ is even, so $P=\left\langle x_{0}, x_{1} \mid x_{0}^{r}=x_{1}^{s}, x_{1}^{r}=x_{0}^{s}\right\rangle$, and let $\alpha, \beta, g$ be as defined in the statement. Then $x_{0}^{\beta r}=x_{1}^{\beta s}=x_{1}^{1-\alpha r}=x_{1} x_{0}^{-\alpha s}$ and hence $x_{1}=x_{0}^{\alpha s+\beta r}$ and so

$$
\begin{aligned}
P & =\left\langle x_{0} \mid x_{0}^{\alpha s^{2}+\beta r s-r}=x_{0}^{\beta r^{2}+\alpha r s-s}=1\right\rangle \\
& =\left\langle x_{0} \mid x_{0}^{\alpha\left(s^{2}-r^{2}\right)}=x_{0}^{\beta\left(s^{2}-r^{2}\right)}=1\right\rangle \cong \mathbb{Z}_{g} .
\end{aligned}
$$

Now $[M: P]=2$ so $|M|=2 g$ and is soluble (regardless of the parity of $q$ ).

Suppose then that $q$ is odd and so $r, s$ are both odd. Then

$$
P=\left\langle x_{0}, x_{1} \mid\left(x_{0} x_{1}\right)^{(r-1) / 2} x_{0}=\left(x_{1} x_{0}\right)^{(s-1) / 2} x_{1},\left(x_{1} x_{0}\right)^{(r-1) / 2} x_{1}=\left(x_{0} x_{1}\right)^{(s-1) / 2} x_{0}\right\rangle,
$$

being an index 2 subgroup of $M$ is soluble of order $g$. The determinant of the relation matrix of $P$ gives $\left|P^{\mathrm{ab}}\right|=2|r-s|$. But $g=|r-s|(r+s)(\alpha, \beta) \geq 3|r-s|$ so $|P| \neq\left|P^{\mathrm{ab}}\right|$ so $P$ is non-abelian. 
Theorem 5.3 Let $N=\left\langle y, t \mid t^{d}=1, y^{s} t^{A}=t^{-A} y^{r}\right\rangle(d \geq 2)$. Then $N$ is large unless one of the following holds:

(a) $A \equiv 0$ mod $d$ and either

(i) $d=2$ and $|r-s|=2$, in which case $N \cong D_{\infty}$; or

(ii) $|r-s|=1$ in which case $N \cong \mathbb{Z}_{d}$.

(b) $(A, d)=1$ and one of the following holds:

(i) $d=2$ and $(r, s)=2$ in which $N$ is infinite and soluble; or

(ii) $r=s=1$, in which case $N \cong \mathbb{Z} \rtimes \mathbb{Z}_{d}$, which is infinite and soluble; or

(iii) $r+s \geq 3,(r, s)=1$ and one of the following holds:

1. $(d,\{r, s\})=(3,\{1,5\})$,

2. $(d,\{r, s\})=(4,\{1,3\})$,

3. $(d,\{r, s\})=(6,\{1,2\})$,

in which case $N$ is infinite and soluble; or

(iv) $r+s \geq 3,(r, s)=1$, and one of the following holds:

1. $d=2$, in which case $N$ is soluble and finite of order $2\left|s^{2}-r^{2}\right|(\alpha, \beta)$, where $\alpha r+\beta s=1$,

2. $(d,\{r, s\})=(3,\{1,2\})$, in which case $N$ is soluble and finite of order 24,

3. $(d,\{r, s\})=(3,\{1,3\})$, in which case $N$ is soluble and finite of order 144,

4. $(d,\{r, s\})=(3,\{1,4\})$, in which case $N$ is insoluble and finite of order 1080,

5. $(d,\{r, s\})=(3,\{2,3\})$, in which case $N$ is insoluble and finite of order 360,

6. $(d,\{r, s\})=(4,\{1,2\})$, in which case $N$ is soluble and finite of order 96,

7. $(d,\{r, s\})=(5,\{1,2\})$, in which case $N$ is insoluble and finite of order 600 .

\section{Proof}

If $d=2$ then the result follows from Theorem 5.2 so assume $d \geq 3$.

(a) If $A \equiv 0 \bmod d$ then $N \cong \mathbb{Z}_{d} * \mathbb{Z}_{|r-s|}$ which is large unless either $d=2$ and $|s-r|=2$, in which case $N \cong D_{\infty}$, or $|s-r|=1$, in which case $N \cong \mathbb{Z}_{d}$.

(b) The group $N$ maps onto $G=\left\langle y, t \mid y^{r+s}=t^{d}=\left(y^{s} t^{A}\right)^{2}=1\right\rangle$. If $\left.(A, d)\right\rangle 1$ then Theorem 5.1 implies that $G$, and hence $N$, is large unless $r=s=1,(A, d)=2$, in which case $N$ maps onto $\left\langle y, t \mid t^{2}\right\rangle \cong \mathbb{Z} * \mathbb{Z}_{2}$, which is large. Suppose then that $(A, d)=1$. By applying an automorphism of $\left\langle t \mid t^{d}\right\rangle$ we may assume $A=1$. If $r=s=1$ then $N \cong \mathbb{Z} \rtimes \mathbb{Z}_{d}$ so assume $r+s \geq 3$. By Theorem 5.1 $G$, and hence $N$ is large unless $(r, s)=1$ and $1 /(r+s)+1 / d \geq 1 / 2$. When we have equality the conditions are equivalent to (b)(iii) and $G$ is infinite and soluble. The cyclic subgroup $H=\left\langle\left(y^{s} t\right)^{2}\right\rangle$ is normal in $N$ and $N / H \cong G$, which is soluble, so $N$ is soluble. When the inequality is strict the conditions are equivalent to (b)(iv) and computations using GAP show that $N$ is finite of the given order and soluble or insoluble as indicated.

This yields

Corollary 5.4 Let $d=(n, A+B)=(n, 2(k-1)-q(r-s))$ and assume $d \geq 2$.

(a) Suppose none of the conditions in Theorem 5.3(a),(b) hold. Then $M(r, n, k, s, q)$ is large. 
(b) Suppose none of the conditions in Theorem 5.3(a)(ii) or (b)(iv) hold. Then $M(r, n, k, s, q)$ is infinite.

Combining the results of this section with those of Section 3 we have

Corollary 5.5 Suppose $(n, A, B) \geq 2,|s-r| \geq 2,(A-B, n) \geq 2$ and $(r, s) \geq 2$. Then $M(r, n, k, s, q)$ is large unless $r=2 R, s=2(R+\epsilon), k=2 K-1, n=2 N$, for some $R, N, K \geq 1, \epsilon= \pm 1$, where $(q, N)=1$ and $(N,(2 K-1)+q \epsilon)=1$.

\section{Proof}

By Corollary 3.8 we may assume $(n, A, B)=|s-r|=(A-B, n)=(r, s)=2$. The conditions $|s-r|=2$, $(r, s)=2$ are equivalent to $r=2 R, s=2(R+\epsilon)$, for some $R \geq 1, \epsilon= \pm 1$. The condition $(A-B, n)=2$ is then equivalent to $n=2 N$ and $(q, N)=1$ for some $N \geq 1$. The condition $(n, A, B)=2$ implies that $k=2 K-1$ for some $K \geq 1$. Applying Corollary 5.4 we see that if $M(r, n, k, s, q)$ is not large then only case of Theorem 5.3 that can hold is (a)(i), and this is equivalent to $(N, 2(K-1)+q \epsilon)=1$.

\subsection{Freiheitssatz methods for Prischepov groups}

In this section we will regard $M(r, n, k, s, q)$ as a one-relator product $(H * K) /<<R>>$ where $\{H, K\}=\left\{\langle x \mid\rangle,\left\langle t \mid t^{n}\right\rangle\right\}, R=y^{s} t^{A} y^{-r} t^{-B}$ where $A=(k-1), B=(k-1)-q(r-s)$. In view of Corollary 3.11 we now investigate when the Freiheitssatz holds in this situation. The following result is contained in [33, Theorem C].

Theorem $5.6([33])$ Suppose $A \not \equiv 0 \bmod n, B \not \equiv 0 \bmod n, 2 A \not \equiv 0 \bmod n, 2 B \not \equiv 0 \bmod n, A \not \equiv$ $\pm B \bmod n$, and $r>2 s$ or $s>2 r$. Then the Freiheitssatz holds for $M(r, n, k, s, q)$ if any of the following hold.

(a) $3 A, 4 A, 5 A \not \equiv 0 \bmod n, B \not \equiv \pm 2 A \bmod n, B \not \equiv-3 A \bmod n, A \not \equiv-2 B \bmod n$;

(b) $3 B, 4 B, 5 B \not \equiv 0 \bmod n, A \not \equiv \pm 2 B \bmod n, A \not \equiv-3 B \bmod n, B \not \equiv-2 A \bmod n$;

(c) $3 A, 3 B \not \equiv 0 \bmod n, B \not \equiv-2 A \bmod n, A \not \equiv-2 B \bmod n$.

In $[35,36,37,38]$ Shwartz considered the Freiheitssatz for one-relator product $(H * K) /<<R>>$ where $R=a b c d \in H * K$ with $a, c \in H, b, d \in K$ and these results can be applied to our situation to obtain other conditions under which the Freiheitssatz holds. We now review Shwartz's results. Let $H_{1}$ be the subgroup of $H$ generated by $\{a, c\}$ and let $K_{1}$ be the subgroup of $K$ generated by $\{b, d\}$. We assume that there are no relations of length 1 or 2 among $\{a, c\}$ in $H_{1}$ or among $\{b, d\}$ in $K_{1}$. By interchanging the roles of $H, K$, cyclically permuting the relator $R$, and replacing $a, b, c, d$ by their inverses we can reduce to the following four cases:

0. $c={ }_{H} a^{ \pm 2}, d={ }_{K} b^{ \pm 2}$;

1. $c={ }_{H} a^{2}, b \neq_{K} d^{ \pm 2}, d \neq_{K} b^{ \pm 2}$;

2. $c={ }_{H} a^{-2}, b \neq_{K} d^{ \pm 2}, d \neq_{K} b^{ \pm 2}$;

3. $c \neq_{H} a^{ \pm 2}, a \neq_{H} c^{ \pm 2}, b \neq_{K} d^{ \pm 2}, d \neq_{K} b^{ \pm 2}$; 
where the subscripts indicate the group in which equality or inequality is considered. Freiheitssatz theorems were obtained by Shwartz for Case 1 in [36], for Case 2 in [37], and for Case 3 in [38]; all of these results are contained in [35]. Case 0 was considered in [17],[18] and our arguments below may be applied to this case; however, since these results are more intricate we limit ourselves to applying the results of Cases 1-3. We summarize Shwartz's results in the following theorem. (In this theorem $A_{4}, S_{4}, A_{5}$ denote alternating and symmetric groups and $Q_{12}$ denotes the quarternionic group of order 12.)

Theorem 5.7 ([35, 36, 37, 38]) Let $G=(H * K) /<<R>>$ where $R=a b c d, a, c \in H, b, d \in K$; let $H_{1}$ be the subgroup of $H$ generated by $\{a, c\}, K_{1}$ be the subgroup of $K$ generated by $\{b, d\}$. Suppose $a \neq_{H} 1, c \neq_{H} 1, a^{2} \neq_{H} 1, c^{2} \neq_{H} 1, a \neq_{H} c^{ \pm 1}, b \neq_{K} 1, d \neq_{K} 1, b^{2} \neq_{K} 1, d^{2} \neq_{K} 1, b \neq_{K} d^{ \pm 1}$, and that $b \neq_{K} d^{ \pm 2}, d \neq_{K} b^{ \pm 2}$. The Freiheitssatz holds in each of the following cases.

1. $c=a^{2}$ and either

(i) $\left|H_{1}\right| \geq 12,\left|K_{1}\right| \geq 10$ and $K_{1} \notin\left\{A_{4}, S_{4}, A_{5}\right\}$; or

(ii) $\left|H_{1}\right| \in\{7,9,10,11\},\left|K_{1}\right| \geq 11$ and $K_{1} \notin\left\{A_{4}, S_{4}, A_{5}\right\}$.

2. $c=a^{-2}$ and either

(i) $\left|H_{1}\right| \geq 9,\left|K_{1}\right| \geq 11$ and $K_{1} \notin\left\{\mathbb{Z}_{12}, A_{4}, S_{4}, A_{5}\right\}$; or

(ii) $\left|H_{1}\right|=7,\left|K_{1}\right| \geq 11$ and $K_{1} \notin\left\{\mathbb{Z}_{12}, A_{4}, S_{4}, A_{5}\right\}$.

3. $c \neq_{H} a^{ \pm 2}, a \neq_{H} c^{ \pm 2}$, and

(i) $H_{1} \notin\left\{A_{4}, \mathbb{Z}_{3} \oplus \mathbb{Z}_{3}\right\}$ and $K_{1} \notin\left\{A_{4}, S_{4}, A_{5}, \mathbb{Z}_{3} \oplus \mathbb{Z}_{3}, \mathbb{Z}_{9}, \mathbb{Z}_{12}, \mathbb{Z}_{15}, Q_{12}\right\}$; or

(ii) $H_{1} \notin\left\{A_{4}, S_{4}, A_{5}, \mathbb{Z}_{3} \oplus \mathbb{Z}_{3}, \mathbb{Z}_{9}, \mathbb{Z}_{12}, \mathbb{Z}_{15}, Q_{12}\right\}$ and $K_{1} \notin\left\{A_{4}, \mathbb{Z}_{3} \oplus \mathbb{Z}_{3}\right\}$.

We may regard $M=M(r, n, k, s, q)$ as a one-relator product $(H * K) /<<R>>$ where $R=a b c d$ in two ways:

(a) $H=\left\langle t \mid t^{n}\right\rangle, K=\langle y \mid\rangle,\{a, c\}=\left\{t^{A}, t^{-B}\right\},\{b, d\}=\left\{y^{s}, y^{-r}\right\}$, and so $H_{1} \cong \mathbb{Z}_{N}, K_{1} \cong \mathbb{Z}$, where $N=n /(n, A, B) ;$ or

(b) $H=\langle y \mid\rangle, K=\left\langle t \mid t^{n}\right\rangle,\{a, c\}=\left\{y^{s}, y^{-r}\right\},\{b, d\}=\left\{t^{A}, t^{-B}\right\}$, and so $H_{1} \cong \mathbb{Z}, K_{1} \cong \mathbb{Z}_{N}$, where $N=n /(n, A, B)$.

By replacing $R$ by $R^{-1}$, inverting generators of $H$ and $K$, and cyclically permuting $R$ we may interchange the roles of $A, B$ and interchange the roles of $r, s$. Therefore in (a) we may take (without loss of generality) $a=t^{A}, b=y^{s}, c=t^{-B}, d=y^{-r}$, and in (b) we may take $a=y^{s}, b=t^{A}, c=y^{-r}, d=t^{-B}$. Applying Theorem 5.7 and then including the cases obtained by interchanging $r, s$ and interchanging $A, B$ we obtain the following theorem. Note that by definition $r \geq 1, s \geq 1$ so many hypotheses are automatic, and note that in (b) Case 1 does not occur. To make clear where each case comes from we keep the numbering here consistent with that of Theorem 5.7.

Theorem 5.8 Suppose $A \not \equiv 0 \bmod n, B \not \equiv 0 \bmod n, 2 A \not \equiv 0 \bmod n, 2 B \not \equiv 0 \bmod n, A \not \equiv \pm B \bmod n$, $r \neq s$ and let $N=n /(n, A, B)$. Then the Freiheitssatz holds for $M(r, n, k, s, q)$ if any of the following hold.

(a) $r \neq 2 s, s \neq 2 r$ and one of the following holds: 
1. $(A \equiv-2 B \bmod n$ or $B \equiv-2 A \bmod n)$ and $N \geq 7, N \neq 8$;

2. $(A \equiv 2 B \bmod n$ or $B \equiv 2 A \bmod n)$ and $N \geq 7, N \neq 8$;

3. $A \not \equiv \pm 2 B \bmod n, B \not \equiv \pm 2 A \bmod n$.

(b) $A \not \equiv \pm 2 B \bmod n, B \not \equiv \pm 2 A \bmod n$ and one of the following holds:

2. $(r=2 s$ or $s=2 r)$ and $N \geq 11, N \neq 12$;

3. $r \neq 2 s, s \neq 2 r$.

Observe that the conditions in b)(3) are the same as a)(3) and that the hypothesis $r \neq s$ can be removed (as in that case killing $t$ shows that $y$ has infinite order). Further, the condition a)(3) does not hold when $N<7$ so we obtain the following tidier formulation.

Theorem 5.8 $\quad$ Let $N=n /(n, A, B)$ and suppose $N \geq 7, A \not \equiv 0 \bmod n, B \not \equiv 0 \bmod n, 2 A \not \equiv 0 \bmod n$, $2 B \not \equiv 0 \bmod n, A \not \equiv \pm B \bmod n$ and that either

(i) $(A \equiv \pm 2 B \bmod n$ or $B \equiv \pm 2 A \bmod n)$ and $r \neq 2 s, s \neq 2 r, N \neq 8$; or

(ii) $A \not \equiv \pm 2 B \bmod n, B \not \equiv \pm 2 A \bmod n$ and if $(r=2 s$ or $s=2 r$ ) then $N \geq 11, N \neq 12$.

Then the Freiheitssatz holds for $M(r, n, k, s, q)$.

Note that for any of the conditions of Theorem 5.6 to hold we require $N \geq 7$ (where $N=$ $n /(n, A, B))$. However, Theorem 5.8' does not generalize Theorem 5.6 since, for example, the group $M(2,16,3,4,1)$ satisfies the hypotheses of Theorem 5.6 but not of Theorem $5.8^{\prime}$.

By Corollary 3.11 we now have

Corollary 5.9 Suppose that the hypotheses of Theorem $5.8^{\prime}$ or Theorem 5.6 hold. Then $M=M(r, n, k, s, q)$ is infinite; moreover,

(a) if $(r, s) \geq 2$ then $M$ contains a free subgroup of rank 2;

(b) if $(A, B, n) \geq 2$ then $M$ is SQ-universal.

Note that for the cases $A \equiv 0 \bmod n$ or $B \equiv 0 \bmod n$ largeness of $P(r, n, k, s, q)$ was completely dealt with in Theorem 4.6 and for the case $A \equiv-B \bmod n$ it was dealt with in Theorem 5.3.

Using Theorem 5.8'(ii) we can obtain a result about Cavicchioli-Hegenbarth-Repovš groups $G_{n}(m, k)$.

Corollary 5.10 Let $N=n /(n, m, k)$ and suppose $N \geq 11, N \neq 12$ and $k \not \equiv 0, k \not \equiv m, 2 k \not \equiv 0$, $2(k-m) \not \equiv 0, m \not \equiv 0, m \not \equiv 2 k, k \not \equiv 2 m, k+m \not \equiv 0,3 k \not \equiv 2 m, m \not \equiv 3 k$ (all mod $n)$ then $G_{n}(m, k)$ is infinite.

It also follows from Theorem $5.8^{\prime}$ that the group $G_{7}\left(x_{0}^{-1} x_{1}^{-1} x_{2}^{-1} x_{4} x_{3} x_{2} x_{1}\right) \cong P(4,7,3,3,1)$ is infinite. This is the one group in [15] that could not be dealt with by computational techniques and required detailed curvature analysis. (Though, of course, Theorem 5.8 relies on the detailed curvature analysis of Shwartz.) In fact, Theorem B of [15], which states that $\left\langle y, t \mid t^{n}, t y^{-3} t^{-2} y^{4}\right\rangle \cong$ $M(4, n, 3,3,1)$ is infinite for all $n \geq 6$ can be recovered as a corollary of Theorem $5.8^{\prime}$ apart from in the cases $n=6,8$. 


\section{The finite Cavicchioli-Hegenbarth-Repovš groups}

Recall that the Cavicchioli-Hegenbarth-Repovš groups $G_{n}(m, k)$ are the groups $P(2, n, k+1,1, m)=$ $G_{n}\left(x_{0} x_{m} x_{k}^{-1}\right)$. Bardakov and Vesnin [2, Question 1] have asked for a classification of the finite groups $G_{n}(m, k)$. With the exception of two unresolved groups the classification has now been obtained. The existing proof of the classification relies on techniques from algebraic number theory [30],[45]. In this section we first review that proof and then build on the results of Section 3 to obtain a new proof that is purely group theoretic.

If $k \equiv 0 \bmod n$ or $(k-m) \equiv 0 \bmod n$ then $G_{n}(m, k)=1$. If $m \equiv 0 \bmod n$ then Corollary 3.5 implies that $G_{n}(m, k)$ is isomorphic to the free product of $(k, n)$ copies of $G_{N}(M, K)$ where $N=n /(n, k)$, $K=k /(n, k)$. By [2, Lemma 1.1(1)] if $K \not \equiv 0 \bmod N$ then $G_{N}(0, K) \cong \mathbb{Z}_{2^{N}-1}$ so $G_{n}(0, k) \cong \mathbb{Z}_{2^{N}-1}$ if $(n, k)=1$ and is infinite otherwise. Thus we may assume $1 \leq m, k \leq n-1, m \neq k$.

Theorem 6.1 ([46]) Let $(n, m, k)=1,(n, k)>1,(n, k-m)>1,1 \leq k, m \leq n-1, k \neq m$ and suppose $G_{n}(m, k) \neq 1$. Then $G_{n}(m, k)$ is finite if and only if $(m, k)=1$ and $(n=2 k$ or $n=2(k-m))$, in which case $G \cong \mathbb{Z}_{s}$ where $s=2^{n / 2}-(-1)^{m+n / 2}$.

The following theorem was proved (in number theoretic terms) in [30] for the case $k=1$ and in [45] for the general case.

Theorem $6.2([30],[45])$ The group $G_{n}(m, k)$ is perfect if and only if either $(m=2 k$ mod $n$ and $(n /(n, m, k), 6)=1)$ or $k=0$ or $m \bmod n$.

Thus we have

Corollary $6.3([45],[46])$ Suppose $(n, k)>1$ and $(n, m-k)>1,1 \leq k, m \leq n-1, k \neq m$. Then $G_{n}(m, k)$ is finite if and only if $(m, k)=1$ and $(n=2 k$ or $n=2(k-m))$, in which case $G_{n}(m, k) \cong \mathbb{Z}_{s}$ where $s=2^{n / 2}-(-1)^{m+n / 2}$.

\section{Proof}

Suppose first that $(n, m, k)=1$. Then $m \neq 2 k \bmod n$ (for otherwise $(n, m, k)=(n, k)>1$ ) so by Theorem $6.2 G_{n}(m, k)$ is not perfect, and hence is not trivial, so the result follows from Theorem 6.1. Suppose then that $d=(n, m, k)>1$. Then by Corollary $3.5 G_{n}(m, k)$ is isomorphic to the free product of $d$ copies of $G_{N}(M, K)$ where $N=n / d, M=m / d, K=k / d$. Since $(N, M, K)=1$ each of these is non-trivial by the above argument, so $G_{n}(m, k)$ is infinite.

By Corollary 4.5 if $(n, k)=1$ or $(n, m-k)=1$ then $G_{n}(m, k)$ is isomorphic to some Gilbert-Howie group $H(n, t)$.

Theorem 6.4 ([23]) Suppose $n \geq 2, t \geq 0,(n, t) \neq(8,3),(9,3),(9,4),(9,6),(9,7)$ and suppose $H(n, t) \neq 1$. Then $H(n, t)$ is finite if and only if $t=0,1$ or $(n, t)=(2 k, k+1)$ where $k \geq 1$ (in which case $\left.H(n, t) \cong \mathbb{Z}_{2^{k}+1}\right)$, or $(n, t)=(3,2),(4,2),(5,2),(5,3),(5,4),(6,3),(7,4),(7,6)$.

We have that $H(n, t)$ is non-trivial by [43, Theorem B] when $t=2$ and by Theorem 6.2 for the case $k=1$ ([30]) otherwise. Moreover the group $H(9,3) \cong H(9,6)$ was proved to be infinite in [12, Lemma 15]. (We remark that the extension of this group also appears in [17, page 228] as $G(-, 9)$.) A calculation in GAP shows that $H(8,3)$ is soluble and of order $3^{10} \cdot 5$. Thus there is the following almost complete classification of the finite groups $H(n, t)$ : 
Corollary 6.5 Suppose $n \geq 2, t \geq 0,(n, t) \neq(9,4),(9,7)$. Then $H(n, t)$ is finite if and only if $t=0,1$ or $(n, t)=(2 k, k+1)$ where $k \geq 1$ (in which case $\left.H(n, t) \cong \mathbb{Z}_{2^{k}+1}\right)$, or $(n, t)=(3,2),(4,2)$, $(5,2)$, $(5,3),(5,4),(6,3),(7,4),(7,6),(8,3)$.

In particular, for $n \geq 10$ there are only finite groups in the families $t=0, t=1$, or $(n, t)=(2 k, k+1)$. Combining Corollary 6.3 and Corollary 6.5 and restricting to the cases $n \geq 10$ we have a classification of the finite groups $G_{n}(m, k)$ :

Corollary 6.6 Suppose $n \geq 10,1 \leq m, k \leq n-1, m \neq k$. Then $G_{n}(m, k)$ is finite if and only if $(n, m, k)=1$ and $(2 k \equiv 0 \bmod n$ or $2(k-m) \equiv 0 \bmod n)$ in which case $G_{n}(m, k) \cong \mathbb{Z}_{s}$ where $s=2^{n / 2}-(-1)^{m+n / 2}$.

Applying Corollary 3.5 we have that $G_{n}(m, k)$ is large whenever $(n, m, k)>1$.

In the next theorem we give a proof of Corollary 6.6 for $n /(n, m, k) \geq 11, n /(n, m, k) \neq 12$ that is purely group theoretic. Since, by Corollary $3.5, G_{n}(m, k)$ is perfect if and only if $G_{N}(M, K)$ is perfect (where $N=n /(n, m, k), M=m /(n, m, k), K=k /(n, m, k)$ ), to verify Theorem 6.2 for $n /(n, m, k) \leq 10$ and $n /(n, m, k)=12$ we may assume $(n, m, k)=1$ and so it suffices to verify it for $n \leq 10$ and $n=12$. This can easily be done by group theoretic methods (for example using GAP). Therefore the proof described above gives a purely group theoretic proof of the classification of the finite groups $G_{n}(m, k)$ for $n /(n, m, k) \leq 10$ and $n /(n, m, k)=12$. This, together with the proof of Theorem 6.7 provides a proof of the (almost complete) classification of the finite groups $G_{n}(m, k)$ that does not involve the algebraic number theory used to prove Theorem 6.2.

Theorem 6.7 Suppose $n /(n, m, k) \geq 11, n /(n, m, k) \neq 12,1 \leq m, k \leq n-1, m \neq k$. Then $G_{n}(m, k)$ is finite if and only if $(n, m, k)=1$ and $(2 k \equiv 0 \bmod n$ or $2(k-m) \equiv 0 \bmod n)$ in which case $G_{n}(m, k) \cong \mathbb{Z}_{s}$ where $s=2^{n / 2}-(-1)^{m+n / 2}$.

\section{Proof}

As in Corollary 6.3 it suffices to prove the result for $(n, m, k)=1$. By Corollary 5.10 we need to consider the cases $2 k \equiv 0,2(k-m) \equiv 0, m \equiv 2 k, k \equiv 2 m, k+m \equiv 0,3 k \equiv 2 m, m \equiv 3 k($ all $\bmod n)$.

If $2 k \equiv 0 \bmod n$ or $2(k-m) \equiv 0 \bmod n$ then $G_{n}(m, k) \cong \mathbb{Z}_{s}$ by [45, Lemma 3]. If $m=2 k$ then $(k, n)=1$ so by Theorem 4.4 we may assume $k=1$ so $m=2$. Then $G_{n}(m, k)=G_{n}(2,1)=S(2, n)$, the Sieradski group. By [43, Theorem B] this is infinite for all $n \geq 6$. If $k=2 m$ then $(m, n)=1$ so by Theorem 4.4 we may assume $m=1$ so $k=2$. Then $G_{n}(m, k) \cong G_{n}(1,2)=F(2, n)$, the Fibonacci group. If $k+m \equiv 0 \bmod n$ then $(k, n)=1$ so we may assume $k=1, m=n-1$ so $G_{n}(m, k)=G_{n}(n-1,1) \cong G_{n}(1,2)=F(2, n)$ by Corollary 4.3. The Fibonacci group $F(2, n)$ is infinite for all $n \geq 9$ (see [42] for a survey of such results).

This leaves the cases $3 k=2 m$ and $m=3 k$. The split extension of $G_{n}(m, k)=P(2, n, k+1,1, m)$ is

$$
M=M(2, n, k+1,1, n)=\left\langle y, t \mid t^{n}=1, y t^{A}=t^{B} y^{2}\right\rangle
$$

where $A=k, B=k-m \bmod n$, and so $(A, B, n)=(n, m, k)=1$. The condition $3 k=2 m$ is equivalent to $A \equiv-2 B \bmod n$ and the condition $3 k=m \bmod n$ is equivalent to $B=-2 A \bmod n$. In the first case we have $(B, n)=1$ so we may assume $B=1, A=-2$; in the second case we have $(A, n)=1$ so we may assume $A=1, B=-2$. Either way we get (by replacing $y$ with $y^{-1}$, if necessary) that $M=\left\langle y, t \mid t^{n}, y^{2} t^{2} y^{-1} t\right\rangle$. This maps onto $L=\left\langle y, t \mid y^{l}, t^{n}, y^{2} t^{2} y^{-1} t\right\rangle$ for any $l \geq 1$. By [18, Theorem 3$]$ if $l \geq 36$ then $y$ has order $l$ in $L$, so $l$ divides $|M|$. Thus $|M| \geq l$ for any $l \geq 36$, so $M$ is infinite. 


\section{References}

[1] J.J. Andrews and M.L. Curtis. Free groups and handlebodies. Proc. Am. Math. Soc., 16:192-195, 1965.

[2] V.G. Bardakov and A.Yu. Vesnin. A generalization of Fibonacci groups. Algebra and Logic, 42(2):131-160, 2003.

[3] Gilbert Baumslag, John W. Morgan, and Peter B. Shalen. Generalized triangle groups. Math. Proc. Camb. Philos. Soc., 102:25-31, 1987.

[4] Gilbert Baumslag and Peter B. Shalen. Amalgamated products and finitely presented groups. Comment. Math. Helv., 65(2):243-254, 1990.

[5] Colin M. Campbell and Edmund F. Robertson. A note on Fibonacci type groups. Can. Math. Bull., 18:173-175, 1975.

[6] Colin M. Campbell and Edmund F. Robertson. On a class of finitely presented groups of Fibonacci type. J. Lond. Math. Soc., II. Ser., 11:249-255, 1975.

[7] Colin M. Campbell and Edmund F. Robertson. On metacyclic Fibonacci groups. Proc. Edinb. Math. Soc., II. Ser., 19:253-256, 1975.

[8] Colin M. Campbell and Edmund F. Robertson. Finitely presented groups of Fibonacci type II. J. Aust. Math. Soc., Ser. A, 28:250-256, 1979.

[9] Colin M. Campbell and Richard M. Thomas. On infinite groups of Fibonacci type. Proc. Edinb. Math. Soc., II. Ser., 29:225-232, 1986.

[10] Alberto Cavicchioli, Friedrich Hegenbarth, and Ann-Chi Kim. A geometric study of Sieradski groups. Algebra Colloq., 5(2):203-217, 1998.

[11] Alberto Cavicchioli, Friedrich Hegenbarth, and Dušan Repovš. On manifold spines and cyclic presentations of groups. In Knot theory, volume 42, pages 49-56. Warszawa: Polish Academy of Sciences, Institute of Mathematics, Banach Cent. Publ., 1998.

[12] Alberto Cavicchioli, E. A. O'Brien, and Fulvia Spaggiari. On some questions about a family of cyclically presented groups. J. Algebra, 320(11):4063-4072, 2008.

[13] Alberto Cavicchioli, Dušan Repovš, and Fulvia Spaggiari. Topological properties of cyclically presented groups. J. Knot Theory Ramifications, 12(2):243-268, 2003.

[14] J. Conway et. al. Solution to advanced problem 5327. Am. Math. Mon., 74:91-93, 1967.

[15] Martin Edjvet. On irreducible cyclic presentations. J. Group Theory, 6(2):261-270, 2003.

[16] Martin Edjvet and James Howie. Intersections of Magnus subgroups and embedding theorems for cyclically presented groups. J. Pure Appl. Algebra, 212(1):47-52, 2008.

[17] Martin Edjvet and Arye Juhász. Equations of length 4 and one-relator products. Math. Proc. Camb. Philos. Soc., 129(2):217-229, 2000.

[18] Martin Edjvet and Arye Juhász. One-relator quotients of free products of cyclic groups. Commun. Algebra, 28(2):883-902, 2000. 
[19] Benjamin Fine, Frank Levin, and Gerhard Rosenberger. Free subgroups and decompositions of one-relator products of cyclics. I: The Tits alternative. Arch. Math., 50(2):97-109, 1988.

[20] Benjamin Fine, Frank Roehl, and Gerhard Rosenberger. The Tits alternative for generalized triangle groups. Baik, Young Gheel (ed.) et al., Groups - Korea '98. Proceedings of the 4th international conference, Pusan, Korea, August 10-16, 1998. Berlin: Walter de Gruyter, 2000.

[21] Benjamin Fine and Gerhard Rosenberger. Algebraic generalizations of discrete groups: A path to combinatorial group theory through one-relator products. Pure and Applied Mathematics, Marcel Dekker. 223. New York., 1999.

[22] The GAP Group. GAP - Groups, Algorithms, and Programming, version 4.4.9, 2006. (http://www.gap-system.org).

[23] N.D. Gilbert and James Howie. LOG groups and cyclically presented groups. J. Algebra, 174(1):118-131, 1995.

[24] George Havas and Edmund F. Robertson. Irreducible cyclic presentations of the trivial group. Exp. Math., 12(4):487-490, 2003.

[25] Graham Higman. A finitely generated infinite simple group. J. Lond. Math. Soc., 26:61-64, 1951.

[26] James Howie. How to generalize one-relator group theory. Combinatorial group theory and topology, Sel. Pap. Conf., Alta/Utah 1984, Ann. Math. Stud. 111, 1987.

[27] D.L. Johnson and H. Mawdesley. Some groups of Fibonacci type. J. Aust. Math. Soc., 20:199-204, 1975.

[28] Arye Juhász. On a Freiheitssatz for cyclic presentations. Int. J. Algebra Comput., 17(5-6):1049$1053,2007$.

[29] K.I. Lossov. The SQ-universality of free products with amalgamated finite subgroups. Sib. Mat. Zh., 27(6):128-139, 1986.

[30] R.W.K. Odoni. Some Diophantine problems arising from the theory of cyclically-presented groups. Glasg. Math. J., 41(2):157-165, 1999.

[31] Stephen J. Pride. The concept of "largeness" in group theory. Word problems II, Stud. Logic Found. Math. Vol. 95, 1980.

[32] Stephen J. Pride. Groups with presentations in which each defining relator involves exactly two generators. J. Lond. Math. Soc., II. Ser., 36:245-256, 1987.

[33] Matvei I. Prishchepov. Asphericity, atoricity and symmetrically presented groups. Comm. Algebra, 23(13):5095-5117, 1995.

[34] Paul E. Schupp. Small cancellation theory over free products with amalgamation. Math. Ann., 193:255-264, 1971.

[35] Robert Shwartz. On the Freiheitssatz in certain one-relator free products with a single relator of length 4. PhD thesis, Technion - Israel Institute of Technology, 1999.

[36] Robert Shwartz. On the Freiheitssatz in certain one-relator free products. I. Int. J. Algebra Comput., 11(6):673-706, 2001. 
[37] Robert Shwartz. On the Freiheitssatz in certain one-relator free products. II. Preprint, 2001.

[38] Robert Shwartz. On the Freiheitssatz in certain one-relator free products. III. Proc. Edinb. Math. Soc., II. Ser., 45(3):693-700, 2002.

[39] Allan J. Sieradski. Combinatorial squashings, 3-manifolds, and the third homology of groups. Invent. Math., 84:121-139, 1986.

[40] Fulvia Spaggiari. Asphericity of symmetric presentations. Publ. Mat., Barc., 50(1):133-147, 2006.

[41] Agnese Ilaria Telloni. Combinatorics of a class of groups with cyclic presentation. Discrete Mathematics, 2010. In press.

[42] Richard M. Thomas. The Fibonacci groups revisited. In Groups, Vol. 2, Proc. Int. Conf., St. Andrews/UK 1989, volume 160 of Lond. Math. Soc. Lect. Note Ser., pages 445-454, 1991.

[43] Richard M. Thomas. On a question of Kim concerning certain group presentations. Bull. Korean Math. Soc., 28(2):219-224, 1991.

[44] Abdullahi Umar. Some remarks about Fibonacci groups and semigroups. Commun. Algebra, 25(12):3973-3977, 1997.

[45] Gerald Williams. The aspherical Cavicchioli-Hegenbarth-Repovš generalized Fibonacci groups. J. Group Theory, 12(1):139-149, 2009.

[46] Gerald Williams. Unimodular integer circulants associated with trinomials. Int. J. Number Theory, 6(4):869-876, 2010. 